\title{
Synthesis and Properties of a Compositional Series of MIL-53(AI) Metal-Organic Framework Crystal-Glass Composites
}

\author{
Christopher W. Ashling ${ }^{1}$, Duncan N. Johnstone ${ }^{1}$, Remo N. Widmer ${ }^{2}$, Jingwei Hou ${ }^{1}$, Sean M. Collins ${ }^{1}$, \\ Adam F. Sapnik ${ }^{1}$, Alice M. Bumstead ${ }^{1}$, Paul A. Midgley ${ }^{1}$, Philip A. Chater ${ }^{3}$, David A. Keen ${ }^{4}$, Thomas \\ D. Bennett ${ }^{1 *}$
}

\author{
${ }^{1}$. Department of Materials Science and Metallurgy, University of Cambridge, Cambridge, CB3 OFS, UK \\ 2. Department of Earth Sciences, University of Cambridge, Downing Street, Cambridge CB2 3EQ, UK \\ ${ }^{3}$. Diamond Light Source Ltd, Diamond House, Harwell Science \& Innovation Campus, Didcot, Oxfordshire, OX11 0DE, \\ UK \\ ${ }^{4}$. ISIS Facility, Rutherford Appleton Laboratory, Harwell Campus, Didcot, Oxon OX11 0QX, UK
}

\begin{abstract}
Metal-organic framework crystal-glass composites (MOF-CGCs) are materials in which a crystalline MOF is dispersed within a MOF glass. In this work, we explore the room temperature stabilisation of the open-pore form of MIL-53(Al), usually observed at hightemperature, which occurs upon encapsulation within a ZIF-62(Zn) MOF glass matrix. A series of MOF-CGCs containing different loadings of MIL-53 were synthesised and characterised using Xray diffraction and nuclear magnetic resonance spectroscopy. An upper limit of MIL-53 that can be stabilised in the composite was determined. The nanostructure of the composites was probed using pair distribution function analysis and scanning transmission electron microscopy. The distribution and integrity of the crystalline component was determined, and these findings related to the MOFCGC gas adsorption capacity in order to identify the optimal loading necessary for maximum $\mathrm{CO}_{2}$ sorption capacity.
\end{abstract}

Metal-organic frameworks (MOFs) are open framework materials, containing metal nodes that are linked in an extended fashion by organic molecules, ${ }^{1}$ that have enabled record breaking surface areas and tunable pore sizes. ${ }^{2,3}$ MOFs are investigated for a plethora of potential applications including; gas storage and separation, catalysis, water harvesting, and sensing. ${ }^{1,4-7}$ The exploitation of the full chemical promise of MOFs in practice may however be impeded by their physical form. Typically, MOFs are synthesised as microcrystalline powders of nm-mm sized particles that are illsuited to industrial settings without prior processing. ${ }^{8}$ This has driven attempts to synthesise bulk materials (monoliths) using techniques such as sol-gel synthesis ${ }^{9-11}$ or post-synthetic compaction and pelletisation via, for example, spark-plasma sintering. ${ }^{12,13}$ Most MOF monolith research has however used a secondary material to aggregate the MOF crystallites such as in MOF-in-silica and mixed-matrix membrane systems. ${ }^{14,15}$

Zeolitic imidazolate frameworks (ZIFs) are a sub-family of MOFs and have proven particularly suitable candidates for the synthesis of MOF monoliths. ZIFs are defined by their incorporation of imidazolate or imidazolate-based linkers, and are noteable amongst MOFs for their high thermal stabilities ( $c a$. 300$\left.500^{\circ} \mathrm{C}\right) .{ }^{16-18}$ The incorporation of these bidentate linkers leads ZIFs to adopt many topologies identical to those of zeolites because the metal-imidazolate-metal dihedral angle is similar to that of siliconoxygen-silicon $\left(\sim 145^{\circ}\right) .{ }^{16}$ Several ZIFs have been observed to undergo melting, forming an extremely viscous MOF liquid at high temperatures, which can be quenched from the melt to form glasses. ${ }^{19}$ Structurally, the glass displays the same short-range order and stoichiometry of the crystalline material, i.e. the metal nodes retain a tetrahedral coordination with a connectivity that can be modelled as a continuous random network (CRN). ${ }^{20}$

The glassy state of MOFs has been used to create MOF crystalglass composites (MOF-CGCs), in which a crystalline phase is incorporated into a MOF-glass matrix. ${ }^{21}$ The first example of such a material was synthesised by forming a physical mixture of crystalline MIL-53( $\mathrm{Al})[\mathrm{Al}(\mathrm{OH})(\mathrm{bdc})]$ (bdc, 1,4-benzenedicarboxylate, $\mathrm{C}_{8} \mathrm{H}_{4} \mathrm{O}_{4}{ }^{2-}$ )- -hereafter referred to as MIL-53 - and ZIF-62 $\left[\mathrm{Zn}(\mathrm{Im})_{1.75}(\mathrm{bIm})_{0.25}\right]$ (Im, imidazolate, $\mathrm{C}_{3} \mathrm{~N}_{2} \mathrm{H}_{3}{ }^{-}$; bIm, benzimidazolate, $\left.\mathrm{C}_{7} \mathrm{H}_{5} \mathrm{~N}_{2}{ }^{-}\right) .{ }^{21}$ This mixture was heated above the melting temperature of ZIF-62, and cooled to room temperature to form the MOF-CGC (MIL-53) $)_{0.25}\left(a_{\mathrm{g}} \mathrm{ZIF}-62\right)_{0.75}$, (i.e. a MOF-CGC com-

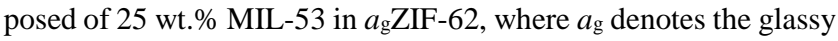
form).

MIL-53 crystallises in a "wine rack" structure (Pnma, $a=$ 17.129(2) $\left.\AA, b=6.628(1) \AA \circ, c=12.182(1) \AA, \alpha=\beta=\gamma=90^{\circ}\right)$ with large internal pores held open by excess solvent and unreacted ligand in the as-synthesised state ("MIL-53-as"). ${ }^{22}$ The removal or replacement of these guest molecules can cause the pore structure to undergo significant volume contraction or expansion, leading to phase transformations at different temperatures (Fig. 1a). When the pore occupying species are expelled, typically by heating, an openpore structure (Imma, $a=6.608(1) \AA, b=16.675(3) \AA, c=$ 12.813(2) $\left.\AA, \alpha=\beta=\gamma=90^{\circ}\right)$ is formed with a greater internal void volume that we refer to as "MIL-53-lp" (large pore). The spontaneous uptake of water into the open-pore phase, typically upon cooling, causes the pores to contract to produce a narrow-pore structure ( $C c, a=19.513(2) \AA, b=7.612(1) \AA, c=6.576(1) \AA, \alpha=$ $\left.\gamma=90^{\circ}, \beta=104.24(1)^{\circ}\right)$ that we refer to as "MIL-53-np" (narrow pore). ${ }^{22}$ Reversible transitions between MIL-53-np and MIL-53-lp are known as "breathing".

It was recently observed that when incorporated into a MOFCGC, the MIL-53-lp structure was stabilised at room temperature (Fig. 1b) and because of the greater pore space compared to MIL53-np, the (MIL-53) $0.25\left(a_{\mathrm{g} Z I F-62)}\right)_{0.75}$ CGC displayed a $\mathrm{CO}_{2}$ uptake greater than a combination of its parent materials. ${ }^{21}$ Motivated by this potential for composite formation to enhance gas sorption and to achieve designed stabilisation of an open pore structure, we study here the maximum loading capacity of MIL-53 within a

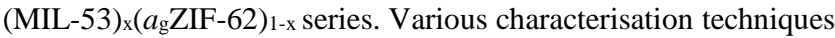
are used to probe the chemical composition and structural integrity of the MOF-CGC components across the (MIL-53) $)_{x}\left(a_{\mathrm{g}} \mathrm{ZIF}-62\right)_{1-\mathrm{x}}$ 
series, and we pay particular attention to the MIL-53 phases present. a)

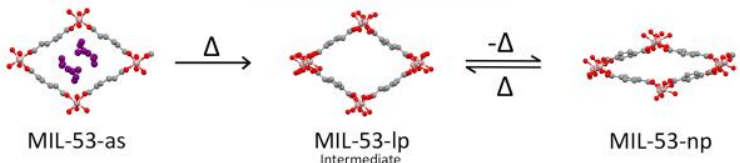

b)
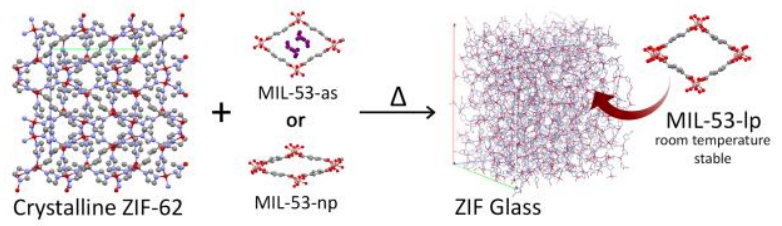

c)
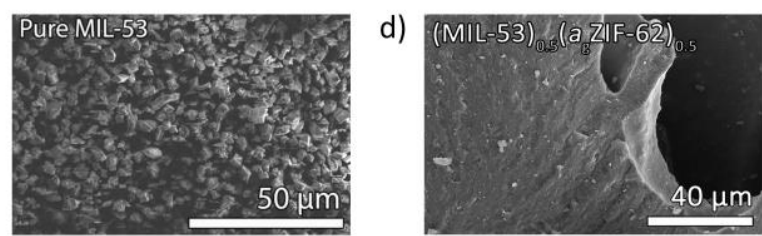

Figure 1. (a) Diagram of the activation process of MIL-53 and the transition between different states $(\mathrm{C}$ - grey, $\mathrm{O}$ - red, $\mathrm{Al}-$ pink, $\mathrm{H}$ - omitted for clarity, solvent - purple); (b) Schematic of composite formation using ball and stick figures of MIL-53, crystalline ZIF62 ( $\mathrm{N}$ - blue, $\mathrm{Zn}$ - red), and stick figure reverse Monte Carlo (RMC) model using combined X-ray and neutron total scattering data of $a_{\mathrm{g} Z I F-} 4^{19}$; (c) Scanning electron microscopy image (SEM) of MIL-53-as - from Hou et al. ${ }^{21}$ (d) Scanning electron microscope (SEM) image of (MIL-53) $0.5\left(a_{\mathrm{g} Z \mathrm{ZIF}-62}\right)_{0.5}$.

\section{RESULTS AND DISCUSSION}

\section{Synthesis of MOF-CGC Series \& Retention of MIL-53-Ip}

Samples of (MIL-53) ${ }_{\mathrm{x}}\left(a_{\mathrm{g} Z \mathrm{ZIF}-62}\right)_{1-\mathrm{x}}$, where $0.3 \leqslant x \leqslant 0.9$ in 0.1 increments, were synthesised by ball milling appropriate masses of crystalline ZIF-62 and MIL-53-as; pressing the resultant wellmixed powder in a $13 \mathrm{~mm}$ diameter dye at $0.74 \mathrm{GPa}$; heating to $450^{\circ} \mathrm{C}$ for 15 minutes; and quenching to room temperature (see Methods). The materials produced were opaque, cream-coloured monoliths, which became brown with increasing concentrations of MIL-53, ascribed to a small amount of thermal decomposition of the MIL-53 component. Neither parent material can be identified from SEM images and the relatively smooth surfaces of the bulk material provide evidence of appreciable flow in the ZIF-62 liquid state (Fig. 1c,d \& S1-S5).

To verify that the stabilisation of MIL-53-lp in (MIL-53) $\left(a_{\mathrm{g}}\right.$ ZIF-

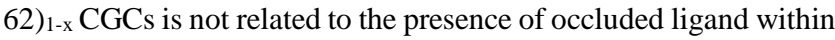
MIL-53-as during CGC formation, a CGC was prepared using MIL-53-np as the starting material. The MIL-53-np was obtained by heating a sample of pure MIL-53-as to $330^{\circ} \mathrm{C}$ for 72 hours, to produce MIL-53-lp which was then cooled to room temperature and underwent the reported phase transition to MIL-53-np (Fig. 1, 2 \& S6). A (MIL-53) $)_{0.25}\left(a_{\mathrm{g}} \mathrm{ZIF}-62\right)_{0.75}$ CGC was then produced (see Methods) using this MIL-53-np starting material and was left in an unsealed container in ambient conditions for 24 hours before labsource powder X-ray diffraction (PXRD) data was collected (See Methods). The experimental pattern displays agreement with the simulated pattern for MIL-53-lp and is dissimilar to MIL-53-np (Fig. 2). This demonstrates that the dominant MIL-53 phase present within (MIL-53) $)_{0.25}\left(a_{\mathrm{g} Z \mathrm{ZIF}} \text {-62) }\right)_{0.75}$ is MIL-53-lp regardless of the starting phase and further composite synthesis reported here were performed using MIL-53-as as the starting material.

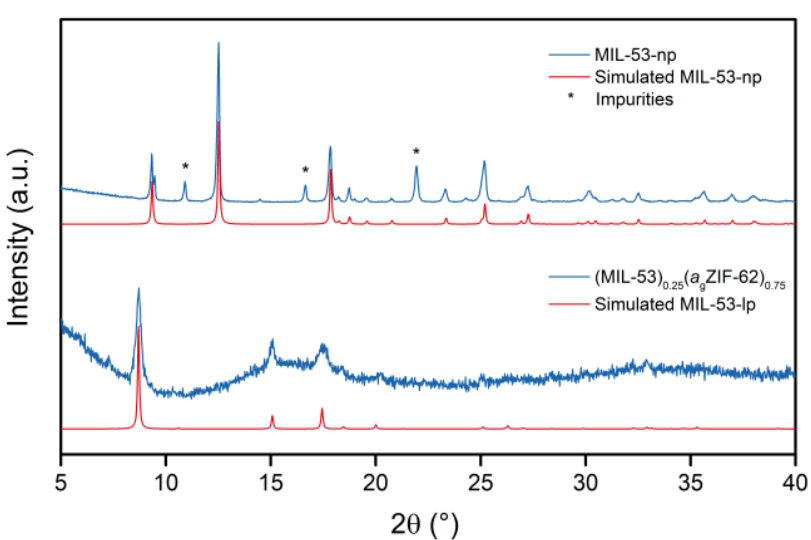

Figure 2. Experimental PXRD patterns (blue) of pure MIL-53-np (top) and the CGC (bottom). The simulated patterns (red) demonstrates the retention of the MIL-53-lp phase in quenched CGCs. Impurities marked with * have also been observed in prior literature. $^{23}$

\section{Composition, MIL-53 Phase Identification, and Loading Ca- pacity}

The integrity and retention of organic linkers in MOF-CGCs across the composition series was studied using solution ${ }^{1} \mathrm{H}$ nuclear magnetic resonance (NMR) (see Methods). The presence of peaks assignable to $\mathrm{HIm}, \mathrm{HbIm}$ and $\mathrm{H}_{2}$ bdc confirmed that the organic linkers present in crystalline ZIF-62 and MIL-53 were also present in all composites (Fig. 3a). A ratio of 1:6.93 between peak intensities corresponding to benzimidazole and imidazole respectively is observed, confirming that there was no change in ZIF-62 stoichiometry upon glass formation (Fig. 3b). The single expected signal arising from $\mathrm{H}_{2}$ bdc is observed to increase, whilst those assigned to HIm and HbIm decrease, as the content of MIL-53 is increased across the MOF-CGC series (Fig. 3a).

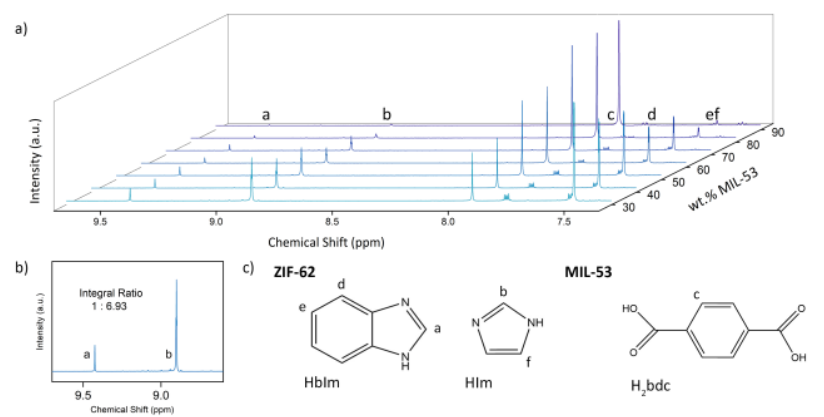

Figure 3. (a) ${ }^{1} \mathrm{H}$ NMR spectra of the (MIL-53) $)_{\mathrm{x}}\left(a_{\mathrm{g}} \mathrm{ZIF}-62\right)_{1-\mathrm{x}} \mathrm{MOF}-$ CGC series, (b) Peak integral ratios of (MIL-53) $)_{0.25}\left(a_{\mathrm{g} Z I F-62}\right)_{0.75}$, (c) Ligands within the frameworks. Peaks in the NMR spectra (a) are assigned (a-f) to protons in the ligands as indicated in (c).

Laboratory PXRD measurements $\left(\mathrm{Cu} K_{\alpha}\right.$ radiation, $\lambda=1.5418$ $\AA$ ) was carried out on the compositional series of finely ground $\left(\right.$ MIL-53) $\left(a_{\mathrm{g} Z I F-62}\right)_{1-\mathrm{x}}$ samples (Fig. 4). The diffraction pattern for (MIL-53) $)_{0.3}\left(a_{\mathrm{g} Z I F-62}\right)_{0.7}$ contained several peaks ascribed to the MIL-53-lp phase, of which the (101), (011), and (202) reflections were most prominent. No reflections were observed which could be ascribed to the MIL-53-np phase. However, upon increasing concentration of MIL-53 within the composite to $70 \mathrm{wt} . \%$, peaks ascribed to the (200) and (110) reflections of MIL-53-np phase 
emerged and increased in relative intensity thereafter. Subsequently, room temperature total scattering data were collected for samples of the (MIL-53) x $_{\mathrm{g}}$ ZIF-62) $)_{1-\mathrm{x}}$ series (and the corresponding crystalline mixtures of the same proportions) using synchrotron radiation ( $\lambda=0.161669 \AA$, see Methods). These data display a rise of a peak emerging at $1.3 \AA^{-1}$ for sample compositions of $70 \mathrm{wt} . \%$ MIL-53 and above (Fig. S7). This peak corresponds to the formation of the MIL-53-np phase and is in agreement with laboratory XRD measurements. For greater phase determination accuracy, Rietveld refinement was performed on the synchrotron-source total scattering data using MIL-53-lp and MIL-53-np crystallographic information files (Table 1, Figs S7-14). ${ }^{22}$ The threshold for MIL53-lp stabilisation, using the materials processing described here, was therefore identified as between 60 and 70 wt.\% MIL-53. Higher incorporations led to the emergence of MIL-53-np.

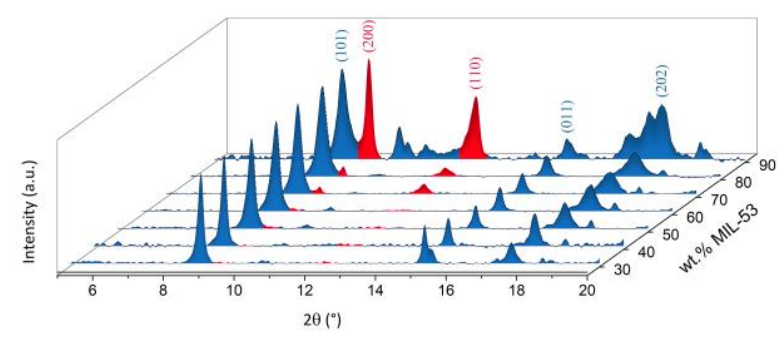

Figure 4. Laboratory powder X-ray diffraction patterns of the (MIL-53) ${ }_{\mathrm{x}}\left(a_{\mathrm{g}} \text { ZIF-62 }\right)_{1-\mathrm{x}}$ composite series, highlighting the MIL-53np Bragg reflections in red. Background subtracted for clarity and normalised to the (101) peak of MIL-53-lp (blue).

\section{Crystal-Glass Composite Microstructure}

Scanning transmission electron microscopy (STEM) was used to investigate microstructure in the (MIL-53) $\left(a_{\mathrm{g} Z I F-62}\right)_{1-\mathrm{x}} \mathrm{CGCs}$ (Fig. 5). Scanning electron diffraction (SED) was used to map the number of Bragg diffraction peaks measured in the diffraction pattern recorded at each probe position, as the electron probe was scanned across the sample, to reveal the location of the crystalline phases in MOF-CGCs, as shown in Fig. 5c \& Figs S15-17. These crystallinity maps demonstrate close contact between crystalline and non-crystalline regions within the MOF-CGCs across the composition range. Comparison with compositional maps showing the distribution of metal centres, obtained via STEM X-ray energy dispersive spectroscopy (STEM-EDS) mapping of the same particles and shown in Fig. 5a \& Figs S15-17, confirms that the crystalline regions correspond to those which are rich in $\mathrm{Al}$ metal-centres, as expected for MIL-53.
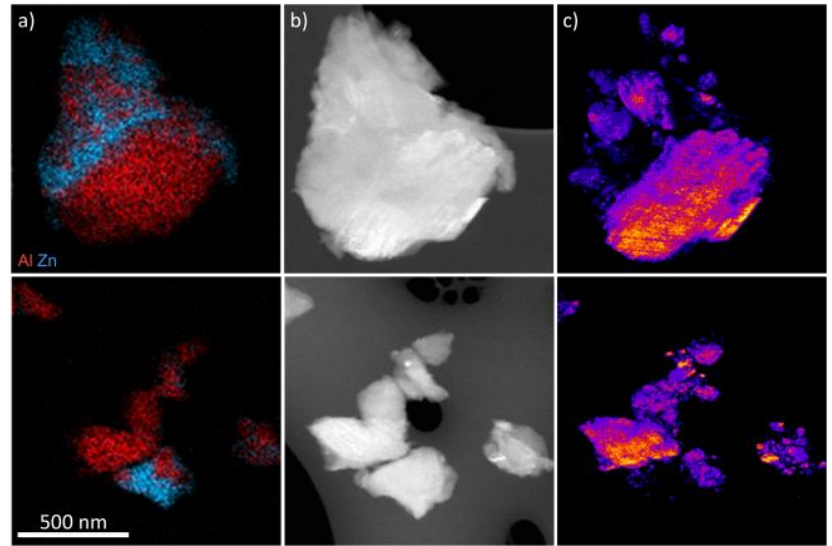

Figure 5. Scanning transmission electron microscopy of MOFCGC particles from (MIL-53)0.6 ( $\left.a_{\mathrm{g} Z I F-62)}\right)_{0.4}$ (upper) and (MIL53) ${ }_{0.9}\left(a_{\mathrm{g} Z I F-62}\right)_{0.1}$ (lower) samples. (a) Compositional maps of $\mathrm{Al}$ and $\mathrm{Zn}$ metal centres from STEM-EDS mapping, (b) Annular darkfield images, and (c) Crystallinity maps showing the number of Bragg peaks as a function of probe position in SED data. Bragg peaks are only recorded from crystalline material and the number of peaks recorded at each position depends on the local crystal orientation. The scale is identical for all images.

Pair distribution function (PDF) analysis is emerging as a powerful tool to investigate interatomic distances in crystalline and amorphous MOFs. ${ }^{24-26}$ This technique yields atom-atom correlation histograms, which effectively indicate distances within a sample and provide information on structure, regardless of crystallinity. Structure factors, $S(Q)$, were obtained from processing the total scattering data of both the (MIL-53 $)_{\mathrm{x}}\left(a_{\mathrm{g} Z I F-62}\right)_{1-\mathrm{x}}$ samples, and the corresponding crystalline mixtures, (MIL-53)(ZIF-62)(X/Y), where $\mathrm{X}$ and $\mathrm{Y}$ represent the respective weight percentages of each component. Those for the (MIL-53)(ZIF-62)(X/Y) series contain the Bragg scattering expected from both ZIF-62 and MIL-53, whereas structure factors from the (MIL-53) $\left(a_{\mathrm{g} Z I F-62}\right)_{1-\mathrm{x}}$ samples

Table 1. Crystallographic data determined from Rietveld analyses of total scattering data of the $(\mathrm{MIL}-53)_{\mathrm{x}}\left(a_{\mathrm{g}} \mathrm{ZIF}-62\right)_{1-\mathrm{x}} \mathrm{X}-\mathrm{ray}$ diffraction series. Rietveld refinement plots are shown in Figs S7-14.

\begin{tabular}{|c|c|c|c|c|c|c|c|c|c|}
\hline & \multicolumn{4}{|c|}{ MIL-53-lp } & \multicolumn{5}{|l|}{ MIL-53-np } \\
\hline Sample & $\begin{array}{c}a \\
(\AA)\end{array}$ & $\begin{array}{c}b \\
(\AA)\end{array}$ & $\begin{array}{c}c \\
(\AA)\end{array}$ & $\begin{array}{c}\text { Quantity } \\
(\%)\end{array}$ & $\begin{array}{c}a \\
(\AA)\end{array}$ & $\begin{array}{c}b \\
(\AA)\end{array}$ & $\begin{array}{c}c \\
(\AA)\end{array}$ & $\begin{array}{l}\beta \\
\left(^{\circ}\right)\end{array}$ & $\begin{array}{c}\text { Quantity } \\
(\%)\end{array}$ \\
\hline MIL-53-Ip* & $6.608(1)$ & $16.675(3)$ & $12.813(2)$ & & & & & & \\
\hline MIL-53-np* & & & & & $19.513(2)$ & $7.612(1)$ & $6.576(1)$ & 104.241(1) & \\
\hline$(\text { MIL-53) })_{0.3}\left(a_{\mathrm{g} Z \mathrm{ZIF}-62}\right)_{0.7}$ & $6.59(1)$ & $16.9(1)$ & $12.65(5)$ & 100 & & & & & 0 \\
\hline$\left(\right.$ MIL-53) $0.4\left(a_{\mathrm{g} Z \mathrm{ZIF}-62)}\right)_{0.6}$ & $6.61(2)$ & $16.7(2)$ & $12.71(9)$ & 100 & & & & & 0 \\
\hline$(\text { MIL-53) })_{0.5}\left(a_{\mathrm{g}} \mathrm{ZIF}-62\right)_{0.5}$ & $6.64(2)$ & $17.0(1)$ & $12.46(6)$ & 100 & & & & & 0 \\
\hline$(\text { MIL-53) })_{0.6}\left(a_{\mathrm{g}} \text { ZIF-62) }\right)_{0.4}$ & $6.64(2)$ & $17.0(1)$ & $12.44(4)$ & 100 & & & & & 0 \\
\hline$(\text { MIL-53) })_{0.7}\left(a_{\mathrm{g}} \text { ZIF-62) }\right)_{0.3}$ & $6.64(2)$ & $16.9(3)$ & $12.55(13)$ & $60(5)$ & $19.22(10)$ & $7.80(4)$ & $6.82(5)$ & 107.5(6) & $40(5)$ \\
\hline$(\text { MIL-53) })_{0.8}\left(a_{\mathrm{g}} \text { ZIF-62) }\right)_{0.2}$ & $6.63(2)$ & $16.8(3)$ & $12.63(11)$ & $55(5)$ & $19.37(11)$ & $7.73(3)$ & $6.91(4)$ & 106.2(4) & $45(5)$ \\
\hline$\left(\right.$ MIL-53) $0.9\left(a_{\mathrm{g} Z I F-62)}\right)_{0.1}$ & $7.02(6)$ & $16.5(1)$ & $12.74(8)$ & $30(5)$ & 19.37(7) & $7.83(2)$ & $6.84(4)$ & $105.8(5)$ & $70(5)$ \\
\hline
\end{tabular}

* Published data by Loiseau et al. ${ }^{22}$ 
contain only that from MIL-53 (Figs S18-26). Appropriate corrections were performed with GudrunX software and the data were Fourier transformed to obtain the corresponding PDFs. ${ }^{27}$

The intensity of the peaks in the PDFs of (MIL-53)(ZIF$62)(\mathrm{X} / \mathrm{Y})$ vary proportionally between the two end members (MIL53-as and crystalline ZIF-62) as a function of the relative proportions of each end member. This is unlike the PDFs of the (MIL$53)_{\mathrm{x}}\left(a_{\mathrm{g} Z I F-62}\right)_{1-\mathrm{x}}$ series which do not display the same ideal conformity between the end members of the series. This is likely due to (i) mixtures of MIL-53-np and MIL-53-lp phases, and (ii) possible interactions at the interfaces between the crystal and glass (Fig. 6). A comparison of the PDFs of (MIL-53)(ZIF-62)(X/Y) and

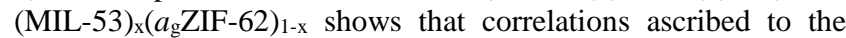
short-range order of ZIF-62 are retained after vitrification (Fig. 6a peaks 1-5). These peaks may be assigned to C-C $(1.38 \AA, \mathbf{1}), \mathrm{Zn}$ -

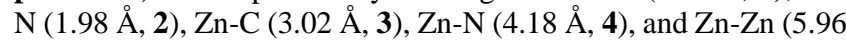
$\AA, 5)$ interatomic distances. In the composites, correlations above $6 \AA$ ascribed to ZIF-62 tend to zero due to the loss of long-range order. Interatomic distances associated with MIL-53 may be identified in both series (Fig. 6a peaks a\&b) - these peaks are assigned to $\mathrm{Al}-\mathrm{C}(4.71 \AA$, a), and $\mathrm{Al}-\mathrm{Al}(6.57 \AA$, b) interatomic distancesthough they are noticeably less intense due to weaker scattering of $\mathrm{Al}$ compared to $\mathrm{Zn}$. In the composite series, all correlations past the short-range order of ZIF-62 ( $~ \AA \AA$ ) originate from crystalline MIL53. Predicted PDF patterns for ZIF-62, MIL-53-as, MIL-53-np, and
MIL-53-lp with their corresponding metal-metal and metal-( $\mathrm{N}$ or O) are provided in Supplementary Figures S27-30.

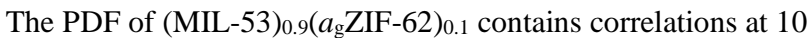
and $14.5 \AA$, which qualitatively agree with peak positions in a predicted PDF for MIL-53-np (Fig. S31). These are absent in the experimental PDF of MIL-53-as. The identification of the MIL-53-np phase within the (MIL-53) $0.9\left(a_{\mathrm{g} Z I F-62}\right)_{0.1}$ agrees with PXRD data collected. We note that the PDFs of (MIL-53) $)_{0.8}\left(a_{\mathrm{g} Z I F-62}\right)_{0.2}$ and (MIL-53) $0.7\left(a_{\mathrm{g} Z I F-62}\right)_{0.3}$ do not display readily distinguishable MIL-53-np PDF correlations belonging to MIL-53-np. This may imply an insufficient concentration of the MIL-53-np phase is present in these samples to give rise to such correlations, especially considering that the peak at $10 \AA$ corresponds to a minimum in the PDFs from MIL-53-lp and MIL-53-as.

\section{Bulk Structure and Properties}

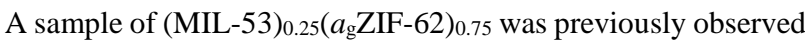
to possess a $\mathrm{CO}_{2}$ gas uptake of $66 \%$ that of a pure sample of MIL53-as. ${ }^{21}$ Given that (i) MIL-53-lp is the main contributor to the adsorption capacity, and (ii) the phase of MIL-53 remains unaltered to loadings of $\leqslant 60 \mathrm{wt} . \%$, the adsorption capacities are expected to increase across those composites displaying only the MIL-53-lp phase. Changes in adsorption trends are however expected on moving to compositions above $60 \mathrm{wt} \%$, i.e. the emergence of the MIL-

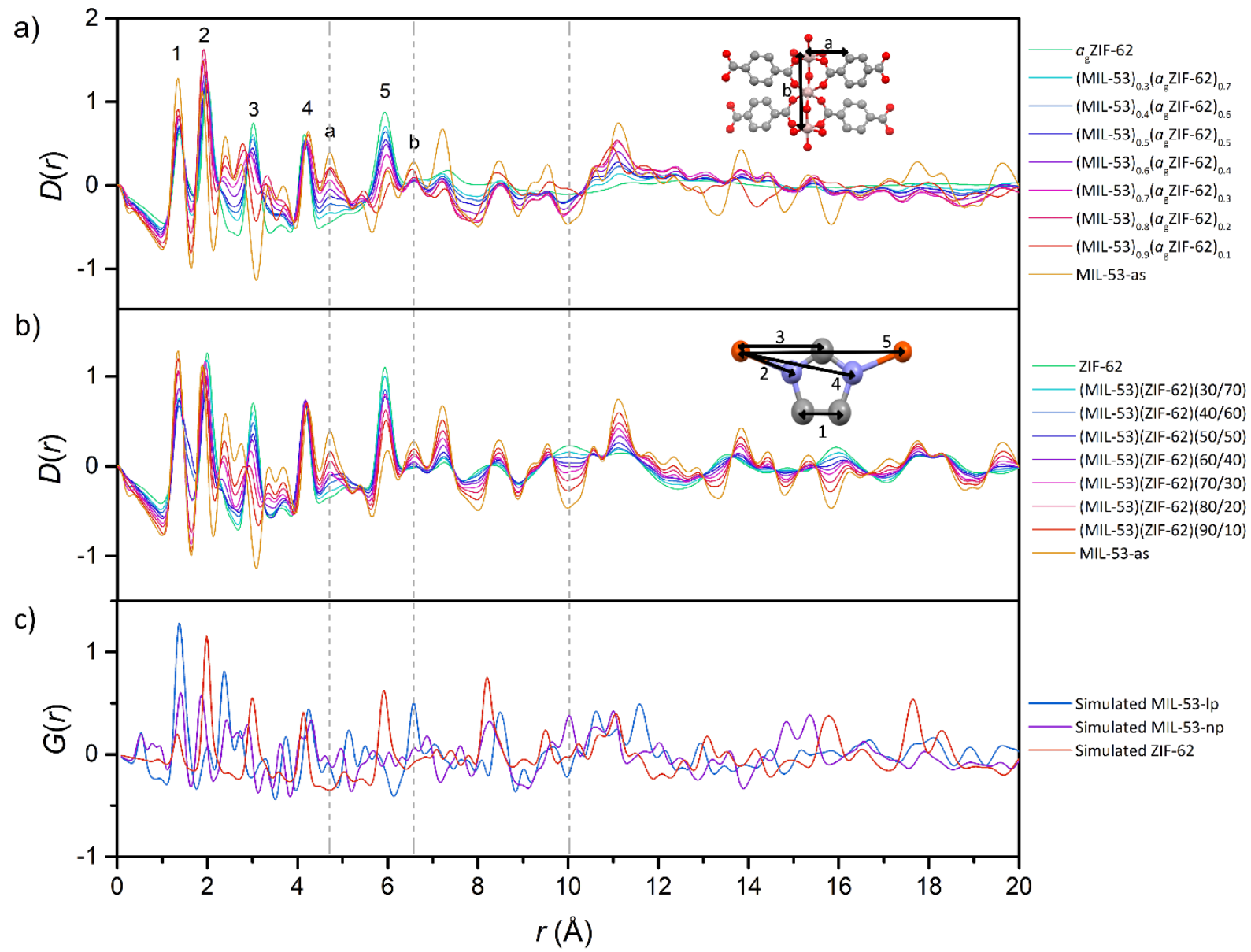

Figure 6. (a) Overlay PDF of the (MIL-53) $\left(a_{\mathrm{g}} \text { ZIF-62) }\right)_{1-\mathrm{x}}$ series with agZIF-62 and MIL-53-as-MIL-53-as and MIL-53-lp are structurally similar and MIL-53-lp is unstable at room temperature. Inset: MIL-53-lp (Al - pink, O - red, C - grey) with correlation assignments (b) Overlay PDF of the crystalline mixtures of the (MIL-53)(ZIF-62)(X/Y) series with MIL-53 and ZIF-62. Inset: ZIF62 ( $\mathrm{Zn}$ - red, N - blue, C - grey) with correlation assignments, and (c) Simulated PDFs of MIL-53-lp, MIL-53-np and ZIF-62 using PDFGUI software. ${ }^{37}$ Sub-1 $\AA$ data due to the way the solvent occupancies were modelled in the published crystallographic information files used. 
53-np phase. The relationship between MIL-53 loading and gas uptake properties was probed using $\mathrm{CO}_{2}$ gas adsorption isotherms for the full compositional series of MOF-CGCs (Fig. 7, for full isotherms see Fig. S32).

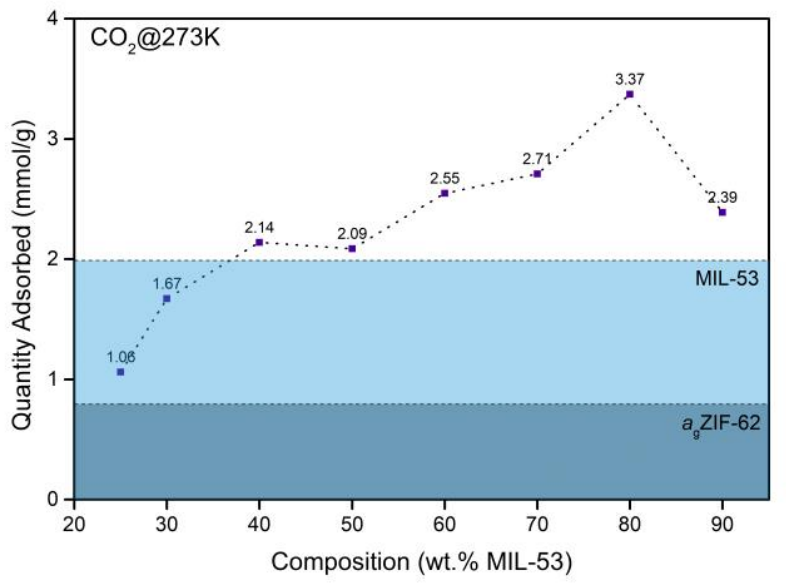

Figure 7. Quantity adsorbed from gas adsorption isotherms for (MIL-53) $\left(a_{\mathrm{g} Z I F-62}\right)_{1-\mathrm{x}}$ series at 1 bar pressure using $\mathrm{CO}_{2}$ gas at

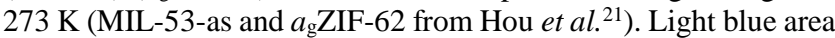
displays the adsorption capacity of MIL-53 and the dark blue area displays that of $a_{\mathrm{g}} \mathrm{ZIF}-62$.

For the samples of (MIL-53) $)_{0.25}\left(a_{\mathrm{g} Z I F-62}\right)_{0.75}-$ (MIL$53)_{0.6}\left(a_{\mathrm{g} Z \mathrm{ZIF}}-62\right)_{0.4}$, the total $\mathrm{CO}_{2}$ quantity adsorbed increases in a broadly linear fashion, from 1.06 to $2.55 \mathrm{mmol} / \mathrm{g}$, in accordance with the increasing concentration of MIL-53 in these samples, which is all present in the MIL-53-lp phase. (MIL-53) ${ }_{0.7}\left(a_{\mathrm{g} Z I F-}\right.$

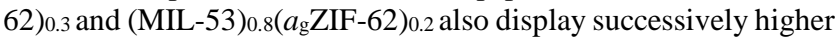
$\mathrm{CO}_{2}$ adsorption. These two samples possess comparable amounts of MIL-53-lp, though the greater proportion of MIL-53 compared to $a_{\mathrm{g} Z I F-62}$ in the latter sample renders its total $\mathrm{CO}_{2}$ uptake higher. A significant decline in capacity is observed for (MIL-53) 0.9 ( $a_{\mathrm{g}} \mathrm{ZIF}$ 62) 0.1 as this material primarily comprises the MIL-53-np phase. Pure samples of MIL-53 and $a_{\mathrm{g} Z I F-62}$ display capacities of 1.99 and $0.79 \mathrm{mmol} / \mathrm{g}$ (at $273 \mathrm{~K}$ at 1 bar pressure) respectively (Fig. 7). As observed by Hou et al. ${ }^{21}$ mesopores are expected in all of the composites.

\section{Conclusion}

In this study we have synthesised a compositional series of (MIL-53) ${ }_{\mathrm{x}}\left(a_{\mathrm{g}} \text { ZIF-62 }\right)_{1-\mathrm{x}}$ crystal glass composites. Sample composition was confirmed by NMR which displayed relevant linker peak integrals varying in proportion to their relative contributions. The crystalline phase contribution of MIL-53 was determined by Rietveld refinement of X-ray diffraction studies - concluding that for MIL-53 loadings up to $60 \mathrm{wt} . \%$, the only phase observed at room temperature is the metastable MIL-53-lp. Beyond this limit, a percentage of the excluded MIL-53-lp phase proceeds to form MIL-53-np upon cooling. This demonstrates a maximum total loading capacity of between $60-70$ wt. $\%$ MIL-53 within $a_{\mathrm{g} Z I F-62}$ in this case. Gas adsorption measurements demonstrate that the maximum effective gas adsorption capacity of the (MIL-53) $\left(a_{\mathrm{g} Z I F-}\right.$ $62)_{1-\mathrm{x}}$ series is around $80 \mathrm{wt} . \%$. Here, the MOF-CGC displays a $\mathrm{CO}_{2}$ adsorption capacity $150 \%$ the value of pure MIL-53-as. A sample of just 30-40 wt.\% loading of MIL-53 is expected to adsorb a similar quantity of $\mathrm{CO}_{2}$ to a sample of pure MIL-53-as. These results show that relatively high loading capacities of crystalline MOFs within a MOF-glass can be achieved and provide a first look at the interesting physical properties which may arise as a result.
It is reasonable to propose that the encapsulation and stabilisation of the metastable states of a range of breathing MOFs is possible, with the expected crystalline phase at room temperature in the composite being that which is stable at the quenching temperature of the matrix glass. We believe that this may open routes to further functional MOF-CGCs and take advantage of many recent reports of other MOF and coordination polymer glass formation. ${ }^{28-31}$

\section{Methods}

Synthesis of MIL-53(Al) Aluminium nitrate nonahydrate $\left(26.00 \mathrm{~g}, 6.93 \times 10^{-2} \mathrm{~mol}\right)$ and terephthalic acid $\left(5.76 \mathrm{~g}, 4.96 \times 10^{-2}\right.$ $\mathrm{mol})$ were dissolved in water $(100 \mathrm{~mL})$ and divided into 5 equal 20 $\mathrm{mL}$ aliquots over 5 Teflon-lined autoclaves. Each reaction vessel was placed in a $220^{\circ} \mathrm{C}$ preheated oven for 72 hours. The white crystalline product was washed with deionised water $(3 \times 30 \mathrm{~mL})$ and dried overnight at $60^{\circ} \mathrm{C} .^{22}$

Synthesis of ZIF-62 Zinc nitrate hexahydrate $\left(3.30 \mathrm{~g}, 1.11 \times 10^{-2}\right.$ $\mathrm{mol})$ and imidazole $(17.82 \mathrm{~g}, 0.262 \mathrm{~mol})$ were dissolved equally in $\mathrm{N}, \mathrm{N}$-dimethylformamide $(150 \mathrm{~mL})$ in two $250 \mathrm{~mL}$ screw top glass jar and stirred for 1 hour. Benzimidazole $(3.10 \mathrm{~g})$ was added to the solutions equally and was stirred for a further hour before closing the jars tightly and placing in an oven preheated to $130^{\circ} \mathrm{C}$ for 48 hours. The solutions were allowed to cool to room temperature before filtering and washing with DMF $(3 \times 30 \mathrm{~mL})$ and DCM $(1 \mathrm{x}$ $30 \mathrm{~mL}$ ) and placed in an oven at $60^{\circ} \mathrm{C}$ to dry overnight to yield a white crystalline powder $(3.26 \mathrm{~g})$, structure confirmed by PXRD. ${ }^{32}$

Synthesis of (MIL-53) $)_{\mathrm{x}}\left(a_{\mathrm{g}} \text { ZIF-62 }\right)_{1-\mathrm{x}}$ MOF-CGCs Crystalline ZIF-62 and MIL-53-as, were balled milled together using a Retsch MM 400 instrument, in appropriate wt. \% ratios using a $7 \mathrm{~mm}$ diameter stainless steel ball for 15 minutes, at a frequency of $30 \mathrm{~Hz}$. The mixed powder was pressed in a $13 \mathrm{~mm}$ diameter dye at 0.74 $\mathrm{GPa}$ for 1 minute. The pellet was then clamped between glass slides, heated to $450^{\circ} \mathrm{C}$ at a rate of $20^{\circ} \mathrm{C} / \mathrm{min}$, and held for 15 minutes, before being allowed to cool to room temperature under an $\mathrm{Ar}$ atmosphere. ${ }^{21}$

X-ray Powder Diffraction Data were collected on ground samples of the composite materials with a Bruker D8 Advance powder diffractometer using $\mathrm{Cu} K_{\alpha}$ radiation $(\lambda=1.5418 \AA)$ and a LynxEye position sensitive detector in Bragg-Brentano $(\theta-\theta)$ parafocussing geometry at room temperature. Diffraction patterns were recorded at $2 \theta$ values of $5-40^{\circ}$ with a time/step of 0.75 seconds over 1724 steps through a $0.012 \mathrm{~mm} \mathrm{Ni}$ filter. PXRD patterns were analysed by Rietveld refinements using TOPAS academic (V6) software. ${ }^{33}$ Pseudo-Voigt shapes were globally refined as a single set of parameters for all scan files. A $9^{\text {th }}$-order Chebychev polynomial background, a Gaussian background peak accounting for the amorphous background from the ZIF-62 glass, scale factors, unit cell parameters of the MIL-53 structure, as well as $8^{\text {th }}$-order spherical harmonics preferred orientation corrections were refined individually for all scans.

Nuclear Magnetic Resonance NMR measurements were carried out by the NMR Service in The Department of Chemistry, University of Cambridge. Data were recorded on a Bruker $500 \mathrm{MHz}$ DCH Cryoprobe Spectrometer. Samples were prepared for NMR by digesting each sample in $0.7 \mathrm{~mL}$ of a premixed solution of $\mathrm{d}^{6}$ dimethyl sulfoxide $(3.5 \mathrm{~mL})$ and deuterium chloride $(1.4 \mathrm{~mL})$, sonicating for 5 minutes and allowing 24 hours for the MOFs to dissolve.

${ }^{1} H$ NMR spectra assignments: ${ }^{1} \mathrm{H}$ NMR (d ${ }^{6}$-DMSO, DCl, 500 $\mathrm{MHz}) \delta_{\mathrm{H}} 7.51(\mathrm{~d}, \mathrm{CCHN}$, Imidazole, $\mathrm{J}=1.3 \mathrm{~Hz}$ ), 7.53 (dd, CCHC, Benzimidazole, $\mathrm{J}=6.2,3.2 \mathrm{~Hz}$ ), 7.81 (dd, CCHC, Benzimidazole, $\mathrm{J}=6.2,3.2 \mathrm{~Hz}$ ), 7.95 (s, Benzene-1,4-dicarboxylic acid, CCHC), 8.90 (t, NCHN, Imidazole, J = 1.25 Hz), 9.43 (s, NCHN, Benzimidazole) 
Fourier Transformed Infra-Red Absorption Samples were finely ground and analysed using a Bruker Tensor 27, scanning wavenumbers of $1-2000 \mathrm{~cm}^{-1}$ over 10 scans (Fig. S33).

Analytical Scanning Transmission Electron Microscopy (STEM) was used to perform crystallinity mapping based on scanning electron diffraction (SED) and compositional mapping based on X-ray energy dispersive spectroscopy (EDS). Data was acquired using a JEOL ARM300F at the Diamond Light Source, UK fitted with a high-resolution pole piece, cold field emitter, and JEOL spherical aberration correctors in both the probe forming and image forming optics. The instrument was operated at $300 \mathrm{kV}$ and aligned in an uncorrected nanobeam configuration and a 10-micron condenser aperture to obtain a convergence semi-angle of $\sim 0.8$ $\mathrm{mrad}$ and a diffraction limited probe diameter $\sim 5 \mathrm{~nm}$. Data was acquired with a scan step size of $\sim 5.2 \mathrm{~nm}$ and a camera length of 20 $\mathrm{cm}$. The probe current was $\sim 2 \mathrm{pA}$. A Merlin-medipix direct electron detector, ${ }^{34,35}$ which is a counting type detector, was used to record the electron diffraction pattern at each probe position with an exposure time of $1 \mathrm{~ms}$ per probe position leading to a total electron fluence of $\sim 5 \mathrm{e} / \AA^{2}$ based on the probe current, exposure time, and assuming a disk-like probe of the diameter above. SED was acquired over a raster pattern comprising $256 \times 256$ probe positions and each diffraction pattern comprised $256 \times 256$ pixels. X-ray energy dispersive spectroscopy (EDS) maps were acquired from the same regions, following SED acquisition, using a larger probe current, obtained using a $150 \mu \mathrm{m}$ condenser aperture, in order to generate sufficient X-ray counts.

SED data were processed using the open source pyXem Python library to find diffraction peaks in every measured diffraction pattern using a difference of Gaussians method, which involves subtracting a blurred version of the diffraction pattern from a less blurred version of the diffraction pattern. EDS data were processed using the open-source HyperSpy Python library to produce maps for each X-ray emission line of interest ( $\mathrm{Al} K_{\alpha}, \mathrm{Zn} K_{\alpha}$ ), which were extracted by integrating an energy window, background subtracted by linear interpolation from adjacent regions of the spectrum without other X-ray peaks present.

Pair Distribution Function Analysis Data were obtained at the I15-1 beamline, Diamond Light Source, UK $(\lambda=0.161669 \AA, 72$ $\mathrm{KeV})$. All samples in both series of (MIL-53) $\left(a_{\mathrm{g} Z I F-62}\right)_{1-\mathrm{x}}$ and (MIL-53)(ZIF-62)(X/Y), along with pure samples of MIL-53-as, ZIF-62 and $a_{\mathrm{g} Z I F-62}$ and both crystalline and glass-ZIF-62 were finely ground before packing into sealed $1.17 \mathrm{~mm}$ (inner) diameter borosilicate capillaries. Data were taken of the background, empty capillary, and of all samples to a $Q_{\max }$ of $26 \AA^{-1}$ with a 10 -minute acquisition time per sample. Normalised total scattering data were corrected individually using the GudrunX program to obtain the PDF of each sample. ${ }^{27,36}$ Predicted patterns were generated using crystallographic information files available online ${ }^{22}$ and the PDFGUI software. ${ }^{37}$

Gas Adsorption $\mathrm{CO}_{2}$ adsorption measurements were performed on a Micromeritics ASAP 2020 instrument at $273 \mathrm{~K}$ (in an ice water bath) using no less than $20 \mathrm{mg}$ of sample. All samples were degassed at $90^{\circ} \mathrm{C}$ for 2 hours followed by $200^{\circ} \mathrm{C}$ for a further 2 hours prior to the adsorption/desorption tests (Fig. S34)

Scanning Electron Microscopy Data were collected on a highresolution scanning electron microscope, FEI Nova NanoSEM 450. Shards of each monolithic MOF-CGC were coated in chromium prior to imaging.

\section{SUPPORTING INFORMATION}

\section{Data Availability}

All data generated in this study are included in this Article and the Supplementary Information, and are also available from the corresponding authors upon request.

\section{Code Availability}

The code used to generate crystallinity maps (Johnstone, D. N. et al., pyXem 0.8.0, 2019) is available at https://doi.org/10.5281/zenodo.2807711. The code used to to produce maps for each X-ray emission line of interest (Peña, F. D. L. et al. hyperspy: HyperSpy $1.4 .1,2019)$ is available at https://doi.org/10.5281/zenodo. 1469364 .

\section{AUTHOR INFORMATION}

\section{Corresponding Author}

Please send any correspondence to tdb35@cam.ac.uk

\section{Author Contributions}

The project was designed by TDB and CWA. PXRD, IR, and gas adsorption measurements were carried out by CWA. SEM measurements were carried out by JH and CWA. PDF measurements were carried out by AFS, AMB, CWA, DAK, PAC and TDB and analysed by CWA and DAK. Rietveld refinements were carried out by RNW and CWA. SMC, DNJ and PAM acquired and analysed STEM data. The manuscript was written by CWA with TDB, with input from all authors.

\section{Notes}

The authors declare no competing financial interests.

\section{ACKNOWLEDGMENTS}

TDB would like to thank both the Royal Society for a University Research Fellowship (UF150021) and the Royal Society for a Research Grant (RG94426). CWA would like to thank the Royal Society for a PhD studentship (RG160498), and the Commonwealth Scientific and Industrial Research Council for additional support (C2017/3108). Both JH and TDB gratefully acknowledge the EPSRC (EP/R015481/1). AFS acknowledges EPSRC for a studentship award under the Doctoral Training Programme. AMB acknowledges the Royal Society for funding (RGFIEA\180092), as well as the Cambridge Trust for a Vice Chancellor's (304253100). We extend our gratitude to Diamond Light Source, Rutherford Appleton Laboratory, UK, for access to Beamline I15-1 (EE20038-1) and access and support in the use of the electron Physical Science Imaging Centre (EM20195). SMC acknowledges the Henslow Research Fellowship at Girton College, Cambridge. PAM thanks the EPSRC for financial support under grant number EP/R025517/1.

\section{REFERENCES}

1 H. Furukawa, K. E. Cordova, M. O’Keeffe and O. M. Yaghi, Science, 2013, 341, 1230444.

2 I. M. Hönicke, I. Senkovska, V. Bon, I. A. Baburin, N. Bönisch, S. Raschke, J. D. Evans and S. Kaskel, Angew. Chemie - Int. Ed., 2018, 57, 13780-13783.

3 O. K. Farha, I. Eryazici, N. C. Jeong, B. G. Hauser, C. E. Wilmer, A. A. Sarjeant, R. Q. Snurr, S. T. Nguyen, A. Ö. Yazaydin and J. T. Hupp, J. Am. Chem. Soc., 2012, 134, 15016-15021.

4 H. Kim, S. Yang, S. R. Rao, S. Narayanan, E. A. Kapustin, H. Furukawa, A. S. Umans, O. M. Yaghi and E. N. Wang, Science, 2017, 356, 430-434.

5 S. Ma and H. C. Zhou, Chem. Commun., 2010, 46, 44-53. 
J.-R. Li, R. J. Kuppler and H.-C. Zhou, Chem. Soc. Rev., 2009 38, 1477-1504

E. Kreno, K. Leong, O. K. Farha, M. Allendorf, R. P. Van Duyne and J. T. Hupp, Chem. Rev., 2012, 112, 1105-1125.

S. Hindocha and S. Poulston, Faraday Discuss., 2017, 201, 113125 .

T. Tian, Z. Zeng, D. Vulpe, M. E. Casco, G. Divitini, P. A. Midgley, J. Silvestre-Albero, J. C. Tan, P. Z. Moghadam and D. Fairen-Jimenez, Nat. Mater., 2018, 17, 174-179.

K. Sumida, K. Liang, J. Reboul, I. A. Ibarra, S. Furukawa and P. Falcaro, Chem. Mater., 2017, 29, 2626-2645.

M. R. Lohe, M. Rose and S. Kaskel, Chem. Commun., 2009, 6056-6058.

R. N. Widmer, G. I. Lampronti, B. Kunz, C. Battaglia, J. H Shepherd, S. A. T. Redfern and T. D. Bennett, ACS Appl. Nano Mater., 2018, 1, 497-500.

M. Rubio-Martinez, C. Avci-Camur, A. W. Thornton, I. Imaz, D. Maspoch and M. R. Hill, Chem. Soc. Rev., 2017, 46, 3453-3480. Q.-L. Zhu and Q. Xu, Chem. Soc. Rev., 2014, 43, 5648-5512.

M. S. Denny Jr., J. C. Moreton, L. Benz and S. M. Cohen, Nat. Rev. Mater., 2016, 1, 16078.

K. S. Park, Z. Ni, A. P. Côté, J. Y. Choi, R. Huang, F. J. UribeRomo, H. K. Chae, M. O'Keeffe and O. M. Yaghi, Proc. Natl. Acad. Sci. U. S. A., 2006, 103, 10186-91.

Y. Q. Tian, Y. M. Zhao, Z. X. Chen, G. N. Zhang, L. H. Weng and D. Y. Zhao, Chem. - A Eur. J., 2007, 13, 4146-4154.

M. Arhangelskis, A. D. Katsenis, N. Novendra, Z. Akimbekov, D. Gandrath, J. M. Marrett, G. Ayoub, A. J. Morris, O. K. Farha, T. Friščić and A. Navrotsky, Chem. Mater., 2019, 31, 3777-3783. R. Gaillac, P. Pullumbi, K. A. Beyer, K. W. Chapman, D. A. Keen, T. D. Bennett and F.-X. Coudert, Nat. Mater., 2017, 16, 1149-1154.

T. D. Bennett, Y. Yue, P. Li, A. Qiao, H. Tao, N. G. Greaves, T. Richards, G. I. Lampronti, S. A. T. Redfern, F. Blanc, O. K. Farha, J. T. Hupp, A. K. Cheetham and D. A. Keen, J. Am. Chem. Soc., 2016, 138, 3484-3492.

J. Hou, C. W. Ashling, S. M. Collins, A. Krajnc, C. Zhou, L. Longley, D. N. Johnstone, P. A. Chater, S. Li, M.-V. Coulet, P. L. Llewellyn, F.-X. Coudert, D. A. Keen, P. A. Midgley, G. Mali, V. Chen and T. D. Bennett, Nat. Commun., 2019, 10, 2580.
T. Loiseau, C. Serre, C. Huguenard, G. Fink, F. Taulelle, M Henry, T. Bataille and G. Férey, Chem. - A Eur. J., 2004, 10, 1373-1382.

W. P. Mounfield and K. S. Walton, J. Colloid Interface Sci., 2015, 447, 33-39.

T. D. Bennett, A. L. Goodwin, M. T. Dove, D. A. Keen, M. G. Tucker, E. R. Barney, A. K. Soper, E. G. Bithell, J. C. Tan and A. K. Cheetham, Phys. Rev. Lett., 2010, 104, 2-5. Billinge,

K. W. Chapman, D. F. Sava, G. J. Halder, P. J. Chupas and T. M. Nenoff, J. Am. Chem. Soc., 2011, 133, 18583-18585.

A. K. Soper, GudrunN and GudrunX: programs for correcting raw neutron and $X$-ray diffraction data to differential scattering cross section. Tech. Rep. RAL-TR-2011-2013 (Rutherford Appleton Laboratory Technical Report, Oxfordshire, 2011). D. Umeyama, S. Horike, M. Inukai, T. Itakura and S. Kitagawa, J. Am. Chem. Soc., 2015, 137, 864-870. Y. Zhao, S. Y. Lee, N. Becknell, O. M. Yaghi and C. A. Angell, J. Am. Chem. Soc., 2016, 138, 10818-10821.

L. Frentzel-Beyme, M. Kloß, R. Pallach, S. Salamon, H. Moldenhauer, J. Landers, H. Wende, J. Debus and S. Henke, J. Mater. Chem. A, 2019, 7, 985-990. Doherty, A. W. Thornton, J. C. Mauro, G. N. Greaves and Y. Yue, Sci. Adv., 2018, 4, eaao6827. Australia, 2007. Crystallogr. Sect. D Biol. Crystallogr., 2013, 69, 1223-1230.

35 J. A. Mir, R. Clough, R. MacInnes, C. Gough, R. Plackett, I Shipsey, H. Sawada, I. MacLaren, R. Ballabriga, D. Maneuski, V. O'Shea, D. McGrouther and A. I. Kirkland, Ultramicroscopy, 2017, 182, 44-53. 714-726.

C. L. Farrow, P. Juhas, J. W. Liu, D. Bryndin, E. S. Boin, J. Bloch, T. Proffen and S. J. L. Billinge, J. Phys. Condens. Matter, 2007, 19, $1-7$. 